\title{
OTIOTOMTCS
}

Revista de economía, empresa y sociedad

NUEVOS PARADIGMAS DE PRODUCCIÓN

\section{Fabricación aditiva y transformación logística: la impresión 3D}

\section{José López Parada}

Profesor colaborador de los Estudios de Economía y Empresa (UOC)

RESUMEN La fabricación aditiva, realizada mediante procesos de impresión 3D, ha tomado gran importancia en algunos sectores de la industria. Esta tecnología, que cuenta actualmente con fuertes planes de investigación e innovación, puede reducir enormemente la complejidad actual en los procesos de fabricación con ventajas adicionales sobre las técnicas de producción convencionales, y también en las actividades logísticas existentes en cada uno de los integrantes de la cadena de suministro.

PALABRAS CLAVE fabricación aditiva; impresión 3D; cadena de valor; cadena de suministro, transformación logística

Additive manufacturing and logistics transformation: the evolution and impact of $3 D$ printing

ABSTRACT Additive manufacturing, performed by 3D printing processes, has gained great importance in certain industrial sectors. Now the subject of significant research and innovation plans, This technology can drastically reduce the current complexity involved in manufacturing processes, with additional advantages over conventional production techniques, as well as in the existing logistics operations at each stage of the supply chain. 
KEYWORDS additive manufacturing; 3D printing; value chain; supply chain; logistic transformation

\section{Introducción}

A lo largo de la historia, la industria ha sufrido grandes revoluciones; a finales del siglo XVIII fue la máquina de vapor la que arranca el proceso con la primera; un siglo después, la disponibilidad de energía eléctrica inicia la segunda; la tercera aparece a mediados del siglo xx con la llegada de la electrónica y la interconectividad mediante internet. En los últimos años, se está iniciando un proceso en el que serán los robots integrados los responsables de una transformación radical, a la cual se la denomina cuarta revolución industrial.

Figura 1. Revoluciones en la industria a lo largo del tiempo

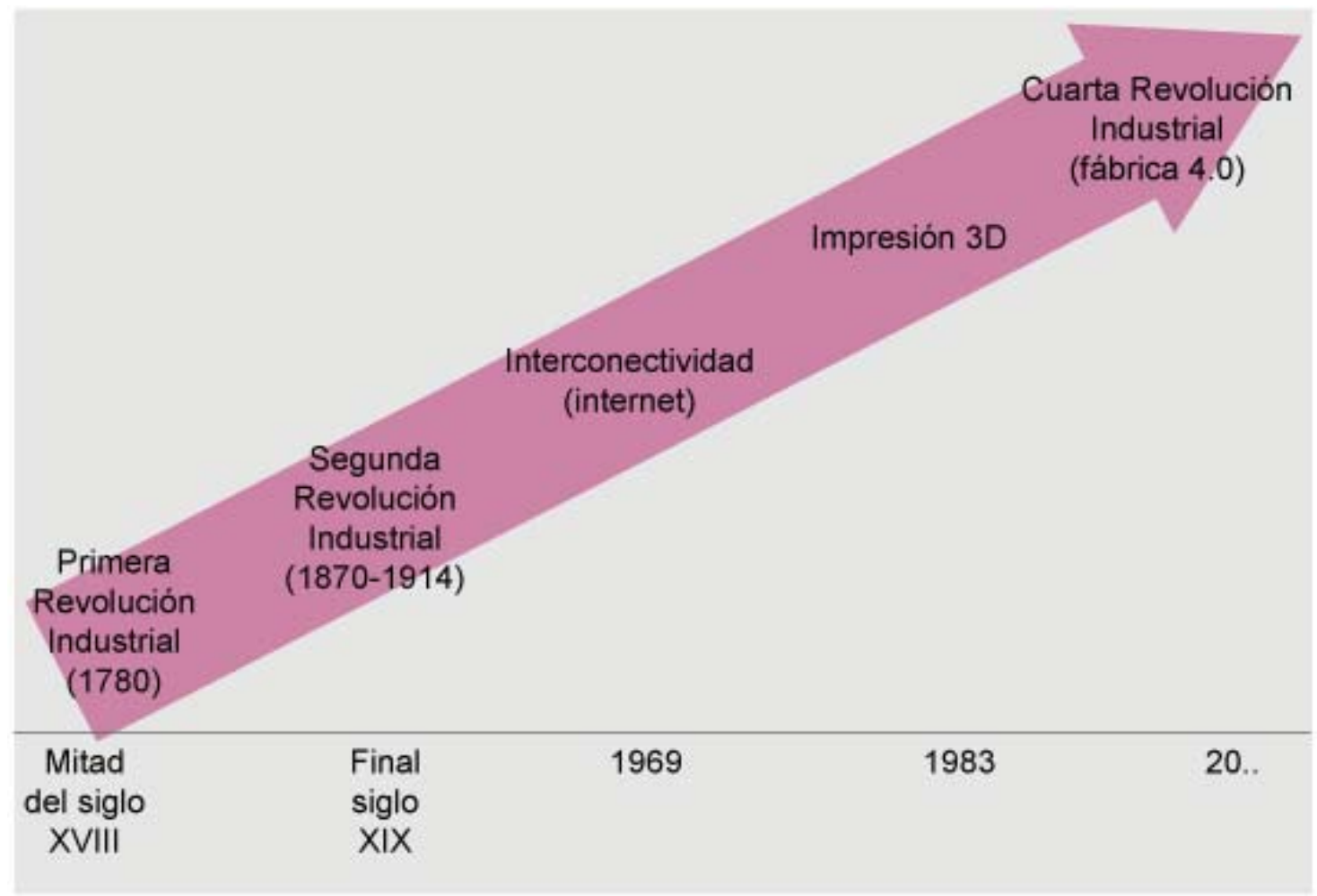

Fuente: elaboración propia.

Es tal la importancia de este tema, que ha sido ampliamente tratado en la reunión del World Economic Forum 2018, que se celebra en Davos.

Vivimos en un mundo donde aparecen constantemente nuevas tecnologías en una evolución permanente: conectividad móvil, internet de las cosas (loT), robótica de inteligencia artificial, así como impresión 3D, materiales avanzados, ingeniería 
genética, nanotecnologías, que, combinadas y conectadas, transformarán los sistemas de producción con una velocidad y un alcance sin precedentes.

En el presente artículo se analiza la transformación logística de la fabricación 3D. Se empieza describiendo un nuevo paradigma en el proceso productivo: la fabricación aditiva, mediante la utilización de tecnologías de impresión en 3D, la situación actual de esta tecnología, su evolución y cómo se materializarán los impactos de la misma en cambios en las cadenas de valor y de suministro de las empresas.

\section{Nuevos paradigmas en producción: la fabricación aditiva}

La primera pregunta que surge es ¿qué es la fabricación aditiva? La fabricación aditiva (en inglés, additive manufacturing), también denominada fabricación por adición, es un nuevo concepto que fusiona la producción en serie y la artesanal, transformando la cadena de suministro y la cadena de valor, lo que implica que la utilización potencial de este modelo productivo afectará de forma importante a la logística empresarial.

Puede definirse como un proceso a través del cual el material, que puede ser plástico o metal, será depositado mediante capas de forma controlada en los puntos en que este es necesario. Con esta técnica, que se conoce comúnmente como «impresión 3D», se fabrican objetos con formas geométricas personalizadas según las necesidades de los clientes.

Si se compara con las técnicas de fabricación tradicionales, esta tecnología modifica el concepto de fabricación, pasando de cortar o moldear las formas, reduciendo o eliminando materiales, al de combinar materias primas de forma más precisa y versátil. Además, al utilizar únicamente el material necesario para la fabricación de la pieza, no se generan deshechos, los componentes tienen un coste menor y se produce de manera más sostenible.

¿Cómo trabaja exactamente la fabricación aditiva? Puede desglosarse en cinco procesos básicos:

1. El primer paso es crear un modelo 3D del objeto que se desea imprimir. Este modelo se realiza mediante un software de diseño (CAD) o mediante técnicas de ingeniería inversa realizando un escaneado del objeto.

2. El fichero CAD se convierte a un formato que defina la geometría de objetos 3D (generalmente, un formato Standard Triangle Language). El archivo se divide digitalmente en capas.

3. La tercera etapa requiere la transferencia del archivo STL y la configuración de la máquina. Para imprimir de manera económica, es decir, maximizar el ahorro de costes y reducir el desperdicio de material, debe verificarse el correcto posi- 
cionamiento y el adecuado tamaño del objeto en la plataforma de construcción. Normalmente, se imprimen varias partes de una vez.

4. En el cuarto paso, la máquina, que únicamente está controlada por el computador, construye el modelo capa a capa. El grosor de cada capa determina la calidad final y depende de la máquina y del proceso.

5. Después de construida la pieza y pasado el período de enfriamiento y seguridad, el modelo se puede quitar de la máquina. Pueden ser necesarios procesos adicionales, como la limpieza, el pulido, la pintura y el acabado de la superficie según el estándar deseado. Esto puede implicar el uso de otras máquinas y herramientas.

Algunas ventajas que ofrece el modelo de fabricación aditiva:

- Mayor capacidad de diseño. La tecnología permite que los ensamblajes puedan imprimirse en un proceso y que las formas orgánicas se puedan producir fácilmente, reduciéndose o eliminando las limitaciones tradicionales de fabricación.

- A diferencia de muchas técnicas de fabricación ampliamente utilizadas, como el moldeo por inyección, no se requieren herramientas.

- Fabricación en cualquier lugar. Las piezas pueden enviarse digitalmente e imprimirse en hogares o lugares cercanos a los consumidores, lo que reduce los requisitos y la dependencia del transporte.

- En comparación con las técnicas convencionales con más limitaciones geométricas, la fabricación aditiva puede producir modelos de forma muy rápida, en horas.

- Utilización de menos recursos para las máquinas y reducida mano de obra cualificada, en comparación con la fabricación convencional.

- Personalización del producto. En especial, es de gran importancia en el sector médico, donde las piezas se pueden realizar directamente para el paciente y sus necesidades individuales.

- Uso eficiente del material debido a la producción exacta de piezas y no sobreproducción en función de la demanda estimada.

- Ventaja comercial y mayor competitividad, en forma de costes y riesgos reducidos, ya que el tiempo de desarrollo, desde el concepto del producto hasta la fabricación del mismo, se reduce al mínimo. La mayor parte del coste es variable, por lo que no se obtienen economías de escala.

- Eficiencia del material, al coincidir el material requerido con el material utilizado. El material de soporte y el polvo, a menudo, se pueden reciclar en la fuente y volver al sistema.

- Beneficios ambientales. Las emisiones derivadas del transporte se reducen debido a la proximidad de fabricación. 
- Con un número cada vez mayor de máquinas, la impresión 3D es cada vez más asequible, mientras que las máquinas de moldeo por inyección siguen siendo relativamente caras e inaccesibles.

\section{Situación actual de la tecnología de impresión 3D}

Existe una conocida curva de maduración tecnológica, denominada Gartner Hype Cycle, que detalla las fases por las que pasa la adopción de una tecnología emergente. En esta curva puede encontrarse un punto de referencia que ayuda a determinar el futuro de la industria de la impresión en 3D.

Figura 2. Ciclo de sobreexpectación de las tecnologías emergentes

\section{Silicon Alley Insider $\quad$ Gráfica del dia}

\section{Ciclo de sobreexpectación de tecnologías emergentes}

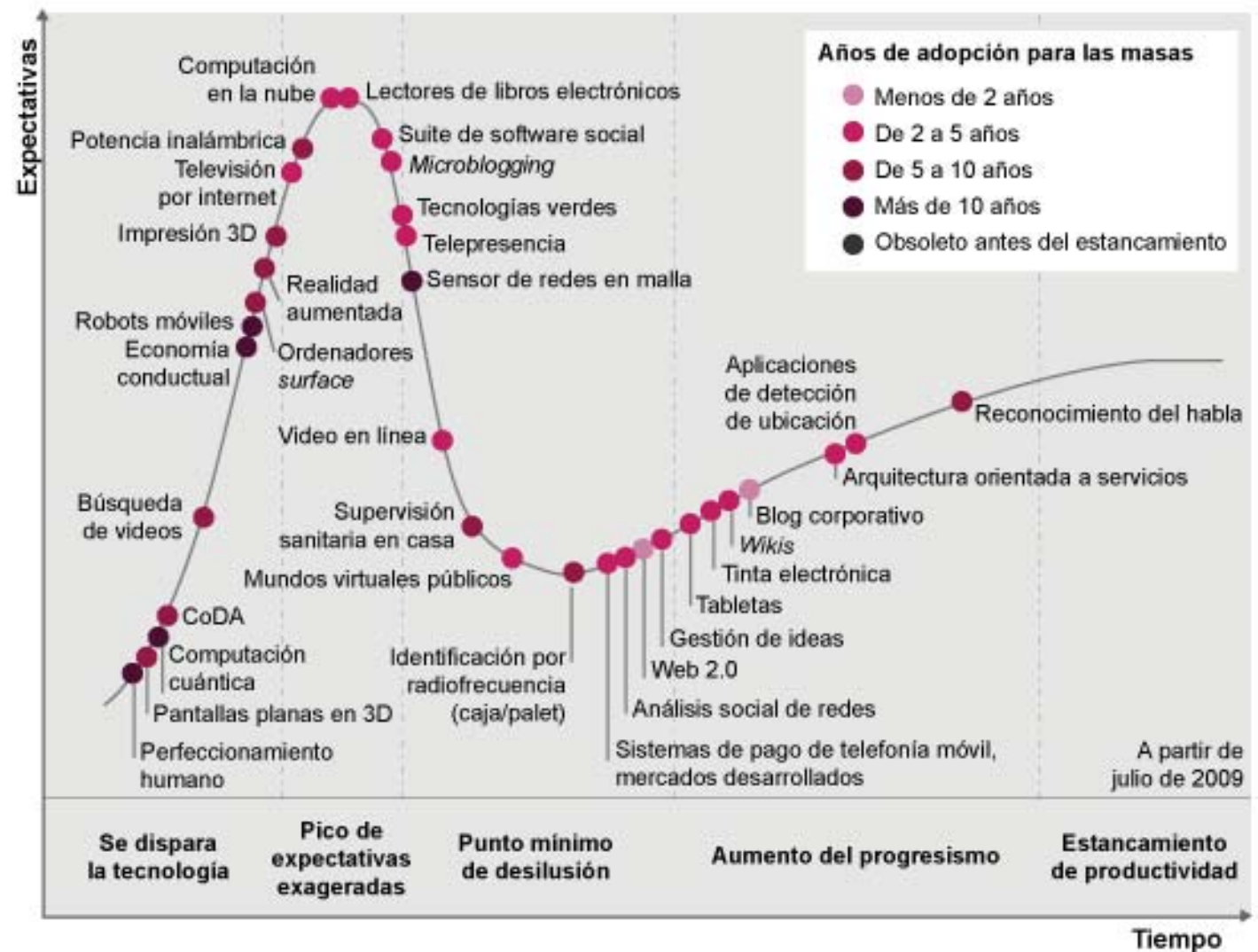

En los inicios, el disparador puede ser un avance tecnológico o una necesidad de mercado. A medida que existe publicidad sobre el tema, se genera un exceso de entusiasmo que se materializa en expectativas poco realistas, aunque pueden 
existir algunas empresas de éxito que utilizan la tecnología, pero no se trata de un modelo generalizado.

El hype continúa creciendo hasta exceder las capacidades actuales y los beneficios de esta tecnología joven pero prometedora; esto, a su vez, crea un «estado de desilusión» en el que los potenciales usuarios la descartan basándose en el hecho de que no ha estado a la altura de las expectativas del mercado. Pero una vez se ha pasado por este abismo de desilusión y las expectativas se restablecen para que coincidan con la realidad, la tecnología puede convertirse en una solución convencional.

La maduración de la impresión 3D se ajusta en gran parte a este ciclo. Concebida para formar productos capa por capa en lugar de mecanizar un bloque de materia prima o realizarlas mediante molde, la impresión 3D permitió la realización de prototipos de una forma más rápida.

La tecnología 3D nació en la década de los ochenta y vivió en relativa oscuridad, alojada en laboratorios de I+D y en talleres de trabajo, durante casi tres décadas. Más adelante, en 2014, comenzó a tener un fuerte desarrollo, especialmente en el sector médico. También, en estos años empezaron a finalizar los periodos de protección derivados de las patentes de los procesos originales de impresión, lo que permitió que llegaran al mercado versiones más pequeñas y ligeras de máquinas industriales, a un precio aceptable para el consumo. Empezaron a surgir experiencias de implantes médicos personalizados y pequeñas piezas en diversos ámbitos profesionales, realizados con equipos de reducido precio (menos de $1.500 €$ ). Sin embargo, las expectativas no fueron todo lo brillantes que se esperaban y el mercado sufrió un proceso de estancamiento.

Actualmente, existe una nueva visión de las posibilidades que ofrece la tecnología 3D en aplicaciones comerciales e industriales, recuperando un nuevo impulso a partir de grandes corporaciones como General Electric (impresora láser más grande del mundo, para impresión mediante polvo de metal) y HP (equipos para entornos de fabricación a escala industrial), que están realizando grandes inversiones en el desarrollo de nuevas soluciones. En pocos meses puede generalizarse la impresión de metales.

Un aspecto a tener muy en cuenta es el actual nivel de confianza. Este se materializa en que las empresas emergentes (startups) de impresión 3D están obteniendo valoraciones muy positivas de la comunidad de empresas de capital riesgo, y lo más importante es que las empresas manufactureras de todos los tamaños están vigilantes a la evolución tecnológica de estas soluciones y la integran en sus planes estratégicos futuros.

En la situación actual, puede observarse una tendencia clave en el mercado, la capacidad de obtener importantes volúmenes de producción con costes aceptables. Puede verse un estudio sobre los costes unitarios según la tecnología utilizada en la figura 3, lo que determinará si realmente estos equipos formarán parte de 
la cuarta revolución industrial. Los procesos productivos precisan de un mayor nivel de velocidad, precisión y confiabilidad, y las nuevas ofertas anunciadas prometen, como mínimo, una velocidad de proceso que permita competir con el moldeado de uretano y por inyección.

Figura 3. Comparación entre dos modelos
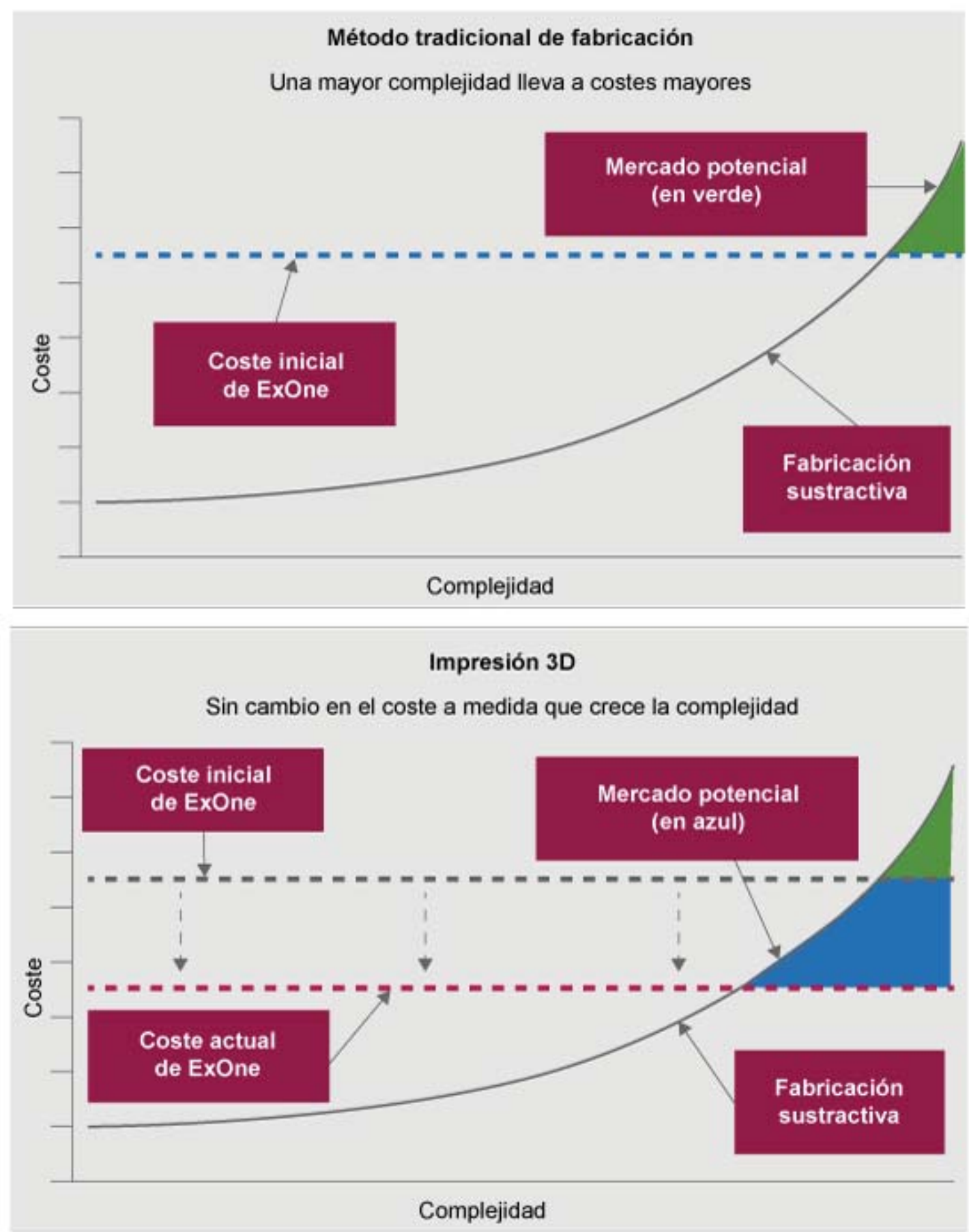

El coste de la máquina por pulgada cúbica se ha reducido un $95 \%$ la última década Los costes por unidad reducidos expanden el mercado potencial de ExOne drásticamente 
Recientemente, HP Development Company, L. P. ha entrado en el mercado con una línea de impresoras denominadas HP Jet Fusion, mediante la impresión de partes funcionales por primera vez al nivel de vóxeles ${ }^{1}$ individuales, y que promete velocidades diez veces más rápidas que las tecnologías de impresión de nailon establecidas, como la extrusión y la sinterización láser. Carbon Inc., una startup respaldada por unos pocos cientos de millones de dólares de capital de riesgo de Silicon Valley, también posee una línea «Carbon SpeedCell ${ }^{\mathrm{TM}}$ » en la que afirma que puede imprimir con velocidades de impresión cien veces mayores que las de las impresoras de resina existentes, y ha declarado públicamente sus intenciones de desplazar el moldeo por inyección con su tecnología. Otras compañías están siguiendo este camino, entre otras: Stratasys, Ltd.; 3D Systems, Inc; EnvisionTEC Inc.; Ricoh Company Ltd.; y Renishaw plc.

\section{Evolución de la tecnología 3D}

La impresión en cuatro dimensiones, también conocida como bioimpresión 4D, origami ${ }^{2}$ activo o sistemas shape-morphing, actualmente se encuentra en periodo inicial de innovación (figura 2 ), utilizando las mismas técnicas de impresión 3D. Sin embargo, la impresión 4D agrega la dimensión de la transformación en el tiempo; tal vez, una mejor manera de expresarlo es que el objeto se transforma con el tiempo.

Es, por lo tanto, un tipo de materia programable, en la que después del proceso de fabricación, el producto impreso reacciona con parámetros de su entorno (humedad, temperatura, etc.) y cambia su forma a partir de los mismos. La capacidad de hacerlo surge de las configuraciones casi infinitas a partir de una resolución micrométrica, creando sólidos con distribuciones espaciales moleculares de alta ingeniería y permitiendo así un alto rendimiento multifuncional. Este tipo de deformaciones estructurales no son nuevas: el conocimiento actual ya ha demostrado propiedades de «memoria» y «material inteligente». Una de las tecnologías más populares se conoce como aleación de memoria de forma, donde un cambio de temperatura desencadena un cambio de forma. Otros enfoques utilizan polímeros

1. Este término se utiliza en el modelado tridimensional, y define la parte distinguible más pequeña de un espacio tridimensional. Cada vóxel, en particular, vendrá identificado por las coordenadas de X, Y y Z de una de sus ocho esquinas, o de su centro. La palabra vóxel está basada en una contracción de la voz VOLUMEN (vox) y de ELEMENTO (el).

2. El origami o papiroflexia es un arte que consiste en el plegado de papel sin usar tijeras ni pegamento para obtener figuras de formas variadas, muchas de las cuales podrían considerarse como esculturas de papel. 
electroactivos, fluidos o gases presurizados, estímulos químicos e incluso respuesta a la luz.

La impresión 4D es un avance relativamente nuevo en la tecnología de la biofabricación, que emerge rápidamente como un nuevo paradigma en disciplinas como la bioingeniería, la ciencia de los materiales, la química y las ciencias de la computación.

\section{Principales impactos de la impresión 3D en la cadena de suministro}

La impresión 3D puede reducir enormemente la complejidad en los procesos de fabricación con ventajas adicionales sobre las técnicas de producción convencionales, y también en las actividades logísticas existentes en cada una de las empresas que configuran la cadena de suministro. A día de hoy, el mayor potencial de la tecnología actual radica en su capacidad para simplificar la producción de productos y componentes complejos y personalizables. En este caso, obliga a redefinir el proceso tradicional de fabricación y suministro.

Las empresas deben analizar y evaluar si los procesos de impresión 3D pueden ser utilizados en su cadena de valor y en la cadena de suministro, pero dado que la impresión 3D implica un rápido desarrollo tecnológico, las inversiones actuales serán obsoletas en pocos años. Por ello, tiene sentido iniciar instalaciones piloto en la obtención de productos selectos, para avanzar posteriormente hacia implantaciones a escala completa, teniendo en cuenta los recursos y capacidades necesarios para obtener «la fábrica digital», mucho más basada en activos lógicos (software) que físicos (utillajes, moldes, herramientas), y por lo tanto mucho más configurable, adaptable y flexible.

En este ámbito, puede incluirse también a las empresas que trabajan mediante estrategias de postponement, ${ }^{3}$ permitiendo niveles más altos en la personalización del producto, algo que tiene gran importancia tanto para los clientes industriales como para los consumidores. Al retrasar el ensamblaje final lo más cerca del punto de demanda, las empresas pueden ofrecer a los clientes acceso a una amplia variedad de opciones de personalización, permitiendo que seleccionen incluso aspectos del diseño, material, forma, tamaño, embalaje y funcionalidades del producto.

3. Estrategia empresarial orientada a retrasar algunas de las actividades de la cadena de suministro hasta que la demanda del cliente se concrete, logrando tanto un mejor control de costes como una mayor velocidad de respuesta a dicha demanda. 
Uno de los puntos que también tiene gran importancia en las empresas actuales es el de las piezas de repuesto, en especial relacionado con los servicios de posventa. En la actualidad, miles de almacenes están ocupados con estas piezas que dan servicio a una gran diversidad de productos. Aunque estos almacenes tienen un gran volumen de movimientos, hay muchos artículos que raramente se precisan (algunos estudios estiman que el exceso de existencias puede estar entre el $20 \%$ y el $25 \%$ ), lo que genera, además de un coste, una gran ineficiencia en la cadena. Una de las soluciones que ofrece esta tecnología es que las empresas no procedan al almacenamiento físico de las piezas de repuesto, sino que se impriman estos componentes bajo demanda, en el lugar que se requieran, lo que permitiría lograr una amplia cobertura, una mayor disponibilidad y un reducido tiempo de entrega, que se traduciría también en una mayor satisfacción para el cliente.

En el ámbito de la logística inversa aparecen otras ventajas al generarse procesos con nulo desperdicio, ya que se consumirá solo el material estrictamente necesario y la energía imprescindible para la fabricación de esos productos, reduciendo los residuos generados y obviamente obteniendo una minoración en las necesidades de transporte y la reducción derivada en emisiones de $\mathrm{CO}_{2}$.

Algunos ejemplos actuales de la impresión 3D en la industria que permiten plasmar las posibilidades que se pueden alcanzar son: el Grupo BMW, que utiliza esta tecnología para la «optimización de topología con impresión 3D, que da a los ingenieros una libertad mucho mayor en la búsqueda de soluciones innovadoras y creativas para el diseño del automóvil»; la empresa Airbus, que anunció en septiembre del pasado año su «primera pieza de titanio impresa en 3D instalada en un avión de producción en serie»; y finalmente, la compañía naviera Maersk Line ${ }^{4}$ que ha equipado a sus buques de carga con impresoras 3D para poder producir a bordo cualquier pieza de recambio que se pueda necesitar durante la travesía.

Además, en el caso de los operadores logísticos, se genera la ventaja de poder conseguir economías de escala, creando una red propia de servicios de impresión 3D, localizada en almacenes y centros de distribución con ámbito global, actuando como un pequeño centro de fabricación (fabshops), de forma que las empresas puedan encargar a sus proveedores logísticos la impresión y la entrega de los pedidos de sus clientes. En esta línea, Amazon ${ }^{5}$ está en proceso de petición de una patente para poder acelerar el reparto de pedidos utilizando camiones de mensa-

4. LNG World Shipping, Maersk and others trial 3D printing on vessels, drilling platforms (15 de diciembre de 2017).

5. Wall Street Journal («When Drones Aren't Enough, Amazon Envisions Trucks with 3D Printers»), 26 de febrero de 2015. 
jería equipados con impresoras 3D que, durante la ruta, imprimen los productos según los piden los clientes.

A pesar de las grandes ventajas expuestas en este artículo sobre la tecnología 3D, deben también considerarse que existen ciertas limitaciones. Una de ellas es el tamaño de las piezas que está en función del propio volumen de los equipos; otra limitación es el tiempo de fabricación que, además del propio necesario en máquina, debido a la alta temperatura en la que se efectúa la impresión, precisa de un tiempo de enfriamiento para la pieza y; finalmente, el elevado precio de las impresoras (entre los 150.000 euros y los 1,5 millones de euros), además del coste de los materiales (entre cuatro y cien veces más caros que los procesos tradicionales).

\section{Conclusiones}

De lo expuesto anteriormente se deriva que el uso de la impresión 3D ha tomado importancia en algunos sectores de la industria, contando además con fuertes planes de innovación tecnológica. El mercado de estas soluciones 3D crecerá rápidamente en los próximos años, pero a pesar de este crecimiento, no se prevé que esta producción sustituya a la producción en masa, sino que más bien se convertirá en un proceso complementario a esta; aunque sí obligará, partiendo de la simplificación, a redefinir las actuales estrategias de fabricación y de la cadena de suministro de las empresas.

\section{Referencias bibliográficas}

BANDYOPADHYAY, A.; BOSE, S. (2015). Additive Manufacturing. CRC press. CHUA, C. (2014). 3D Printing and Additive Manufacturing. World Scientific Publishing.

GARTNER (2018). Top Trends in the Gartner Hype Cycle for EmergingTechnologies, 2017. [Fecha de consulta: 8 de enero de 2018]. <https://www.gartner. com/smarterwithgartner/top-trends-in-the-gartner-hype-cycle-for-emergingtechnologies-2017/>

GEBHARDT, A. (2012). Understanding Additive Manufacturing: Rapid Prototyping, Rapid Tooling, Rapid Manufacturing. Hanser Gardner Publications.

LIPSON, H.; KURMAN, M. (2015). La revolución de la impresión 3D. Anaya.

SCHWAB, K. (2016). La cuarta revolución industrial. Penguin Random House.

WORLD ECONOMIC FORUM (2018). Cuarta revolución industrial. [Fecha de consulta: 25 de enero de 2018). <https://www.weforum.org/es/agenda/archive/ fourth-industrial-revolution/> 


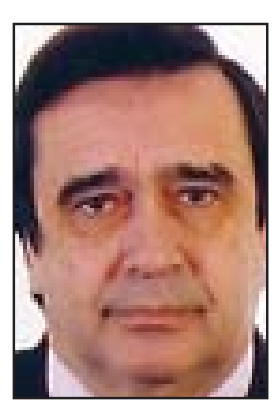

\section{José López Parada}

jlopezpar@uoc.edu

Profesor colaborador de los Estudios de Economía y Empresa (UOC)

Doctor por la Universidad de Barcelona, licenciado en Ciencias Económicas y Empresariales, ingeniero industrial por la UPC, diplomado por el IESE. Exprofesor del Departamento de Empresa de la Facultad de Economía y Empresa de la Universidad de Barcelona. Exprofesor del grado de Ingeniería de Organización Industrial de la Escuela Universitaria Salesiana de Sarria, adscrita a la Universidad Autónoma de Barcelona. Ha realizado tareas directivas, entre otras, en las siguientes empresas: Softmatic (managing director), Digital Equipment Corporation (director de proyectos de alto riesgo) y grupo Philips (director de organización).

Los textos publicados en esta revista están sujetas -salvo que se indique el contrario- a una licencia de Reconocimiento 3.0 España de Creative Commons. Podéis copiarlos, distribuirlos, comunicarlos públicamente y hacer obras derivadas siempre que reconozcáis los créditos de las obras (autoría, nombre de la revista, institución editora) de la manera especificada por los autores o por la revista. La licencia completa se puede consultar en http://creativecommons.org/licenses/by/3.0/es/deed.ca.

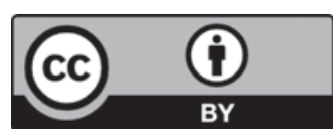

